\title{
Shape Recognition Using Combined Moment Invariants and PCA
}

\author{
Hyeonseok Jeong and Sungyoung Kim ${ }^{1}$ \\ Dept. of Computer Engineering, Kumoh National Institute of Technology, \\ Daehak-ro 61, Gumi, Gyeongbuk 730-701, Korea \\ asdfgh25@naver.com,sykim@kumoh.ac.kr
}

\begin{abstract}
In this paper, we combine two moments of $\mathrm{Hu}$ and Zernike to try improve the recognition accuracy. And we apply PCA on the Zernike moments to speedup of recognition without degrading the recognition accuracy. We compare the recognition performance between $\mathrm{Hu}$ and Zernike moments. Also we show the recognition accuracy of new methods with two modified features. We evaluate the recognition accuracy with two datasets of hands and digits. For hand recognition, we localize hands from hand candidates with improved method against our preliminary studs, [2].
\end{abstract}

Keywords: moment invariants, hand shape reoghition, digit recognition, PCA, skin color segmentation, vehicle license plate

\section{Introduction}

Shape and gesture recognition is andmportant initastep in human-device interaction (HDI) which is a key part of intel igent computer vision applications. Human-device interaction is more general term than human-computer interaction (HCI). Technologies about HDI evolve rapidly to increase converience of device control for humans. Human body may be more helpful and suitable to control device than some operating devices such as mouse or remote controllers that are conventional devices.

Some software libraries equipped with powerful camera systems such as Kinect or Leap motion senson shows excellent performance. The libraries, however, must be coupled with canera system and ate not only quite expensive against usual web-cam but also a big size to embed in other devices such as smart electric whiteboard system.

It's very ordinary to dtilze shape and gesture recognition in our life. There are some examples. Gesture recognition is used in smart TV for remote control. Digits recognition in license plates is for unmanned billing center in parking lots. Smart electric whiteboards for remote control and drawing at long distance apart from location of whiteboards need hand and gesture detection [1]. These techniques are useful in robot control and entertainments [2].

A lot of research on shape descriptors have been studied and in progress. Some of them give good performance in many applications concerned with pattern recognition and computer vision. A moment is a quantitative measure that capture the shape or significant features of a set of points.

Moments are used intensively for many computer vision applications. They are used for static hand gesture recognition [2-4] or for dynamic gesture [5]. They are also used for other shape recognition such as Chinses characters [6, 7], iris [8], faces [9] and traffic signs [10]. Many studies on moments show that moments are very suitable for object recognition. There was some research to speedup calculation in moments [11, 12].

* Corresponding author: Dept. of Computer Engineering, Kumoh National Institute of Techonogy, Daehak-ro 61, Gumi, Gyeongbuk 730-701, Korea (sykim@kumoh.ac.kr) 
In this paper, we compare the recognition performance between $\mathrm{Hu}$ and Zernike moments. There are some studies to recognize hand gestures by using $\mathrm{Hu}$, Zernike, Tchebichef or Krawtchouk [2-4]. We combine two moments of Hu and Zernike to try improve the recognition accuracy. And we apply PCA on the Zernike moments to speedup of recognition without degrading the recognition accuracy.

We use two kinds of dataset for evaluating the performance of recognition, first one consists of hand and another includes digits from vehicle license plates. For hand shape recognition, we segment hand regions from input images. Hand regions are segmented based on skin color detection and remove all unnecessary segmented regions except hand candidates. In this processing step, we remove faces from the segmented regions based on skin colors based on LBP cascades that is modified version of Haar cascades [13]. By the way, a hand candidate can be consisted of not only hand but also wrist or forearm connected with the hand. So we localize hands from hand candidates with advanced method against our preliminary study [2]. We show intensive evaluation results on recognition about two dataset.

This paper consists of three parts. We introduce theory of moments and characteristics on Zernike moments in chapter 2. In chapter 3, we suggest an extraction method of hand candidates and a new localization method of hands from hand candidates Then we show intensive evaluation results on recognition and conclude our study.

\section{Moment Invariants}

A moment is a quantitative measure that capture the shape or significant features of a set of points. Geometric moments $m_{p q}$ of an Tnage are defined as in Eq. (1) where $r=p+q$ is called an order of the moment. $x^{p} y^{q}$ is a basis function for geometric moments.

$$
m_{p q}=\Sigma_{x x} \Sigma q^{p} y^{q} f(x,
$$

Most objects in the images change their appearance with translation, scaling and rotation. So invariant representation of objects with respect to the transformations is very important. Geometric moments, however are dependent on the transformations. Central moments and normalized central moments are used for invariance with respect to translation, and translation and uniform sealing, respectively. Central geometric moments and normalized centrar geometric moments are defined as in Eq. (2) and (3). $\bar{x}$ and $\bar{y}$ are centrode of the pbject. In this paper, we localize the location of extracted objects with minimum bounding rectangle, and so we don't care for translation invariance but should consider scale and rotation inyariance.

$$
\begin{gathered}
\mu_{p q}=\sum_{x} \sum_{y}(x-\vec{x})^{p}(y-\vec{y})^{q} f(x, y) \\
\eta_{p q}=\frac{\mu_{p q}}{\left(\mu_{00}\right)^{Y}}, \text { where } y=\left\lfloor\frac{p+q}{2}\right\rfloor+1
\end{gathered}
$$

$\mathrm{Hu}$ [3] introduced new moment invariants that are invariant to in-place rotation around the origin $\mathrm{Hu}$ moments are also invariant to translation and scaling. Hu's moment invariantsare defined as in Eq. (4).

$$
\begin{gathered}
\varphi_{1}=m_{20}+m_{02} \\
\varphi_{2}=\left(m_{20}-m_{02}\right)^{2}+4 m_{11}^{2} \\
\varphi_{3}=\left(m_{30}-3 m_{12}\right)^{2}+\left(3 m_{21}-m_{03}\right)^{2} \\
\varphi_{4}=\left(m_{30}+m_{12}\right)^{2}+\left(m_{21}+m_{03}\right)^{2} \\
\varphi_{5}=\left(m_{30}-3 m_{12}\right)\left(m_{30}+m_{12}\right)\left(\left(m_{30}+m_{12}\right)^{2}-3\left(m_{21}+m_{03}\right)^{2}\right) \\
+\left(3 m_{21}-m_{03}\right)\left(m_{21}+m_{03}\right)\left(3\left(m_{30}+m_{12}\right)^{2}-\left(m_{21}+m_{03}\right)^{2}\right), \\
\varphi_{6}=\left(m_{20}-m_{02}\right)\left(\left(m_{30}+m_{12}\right)^{2}-\left(m_{21}+m_{03}\right)^{2}\right) \\
+4 m_{11}\left(m_{30}+m_{12}\right)\left(m_{21}+m_{03}\right) \\
\varphi_{7}=\left(3 m_{21}-m_{03}\right)\left(m_{30}+m_{12}\right)\left(\left(m_{30}+m_{12}\right)^{2}-3\left(m_{21}+m_{03}\right)^{2}\right) \\
+\left(m_{30}-3 m_{12}\right)\left(m_{21}+m_{03}\right)\left(3\left(m_{30}+m_{12}\right)^{2}-\left(m_{21}+m_{03}\right)^{2}\right)
\end{gathered}
$$


Regular moments including Hu's moments have some problems [14]. First of all, the basis set $x^{p} y^{q}$ is not orthogonal and so the moments contain redundant information. And as order of $x^{p} y^{q}$ increases, high computing power and time is required. We can overcome these issues by using orthogonal moments that use orthogonal basis sets. Orthogonal moments require lower computational power against regular. There are several orthogonal moments such as Zernike, Tchebichef and Krawchouk. Zernike moments are continuous but Krawchouk moments discrete. Continuous moments can have discretization error but Zernike moments are very common in computer vision applications.

Zernike moments [14] are defined as Eq. (5) which project $f(x, y)$ onto orthogonal basis function $V_{n m}(x, y)$ where $n$ is called order and m repetition, and $x^{2}+y^{2} \leq 1$.

$$
A_{n m}=\frac{n+1}{\pi} \sum_{x=0}^{N-1} \sum_{y=0}^{M-1} f(x, y)\left[V_{n m}(x, y)\right]^{*}
$$

The basis function $V_{n m}(x, y)$ is defined as Eq. (6) and (7). $R_{n m}(x, y)$ is called a radial polynomial and $n \in N^{+}, m \in N,(n-|m|)$ is even, $|m| \leq n$

$$
R_{n m}(x, y)=\sum_{s=0}^{(n-|m|) / 2}(-1)^{s} \frac{(n-s) !}{s !\left(\left(\frac{n+|m|}{2}-s\right) !\right)\left(\left(\frac{n-|m|}{2}-s\right) !\right)}\left(x^{2}+y^{2}\right)^{(n-2 s) / 2}
$$

Zernike moments are orthogonal moments that are defined on a disk space. Locations of points in disk space use polar coordinate system that rêpresents locations of points with radial factor and angular factor. Moments on a disk have an advantage that they can achieve rotation invariants more easily than moments or rectangle.

The basis function and the radial bolynomial of zernike moments in polar coordinate system can be changed as Eq. (8) and (9). So the radial polynomial in polar coordinate system is called as orthogonal radial polynomial.

$$
\begin{aligned}
& V_{n m}(\rho, \theta)-R_{n m}(\rho) e^{j m \theta} \\
& \text { where } \rho=\sqrt{x^{2}+y^{2}}, \theta=\tan ^{-1}\left(\frac{y}{\chi}\right) \\
& (n-s) ! \\
& s !\left(\left(\frac{n+|m|}{2}-s\right) !\right)\left(\left(\frac{n-|m|}{2}-s\right) !\right)
\end{aligned} \rho^{(n-2 s)}
$$

\section{Recognition of Hand Shapes}

\subsection{Extraction of Hand Regions}

First step in recognition of hand shapes is to detect hand candidates from input images. We segment hand candidates based on skin colors in the images. Color, however, is not so robust to extract hand candidates because color can be changed with lighting conditions and human race. Skin colors can be segmented if we use the skin color segmentation method such as in [15] and [16]. And it's possible to use multiple proposal for and detection [17]. These methods, however, need much computation time and so are not adequate for real-time applications.

We extract pixels with colors within predefined range of bins in 2D histogram of hue $(\mathrm{H})$ and saturation $(\mathrm{S})$ in HSV color space to select hand candidates [2]. Sometimes faces can be one of hand candidates because faces has similar color distribution with hands. We try to remove faces from hand candidates based on face detection using LBP cascades. 
Face can be detected with Haar and LBP cascades. Detection with Haar cascade is better than LBP cascades in accuracy but slower in processing time. So we use face detection method using LBP cascades. After all, some post processing methods such as morphological operation are performed. Figure 1 shows an example of skin color detection and the result of removing a face from hand candidates.
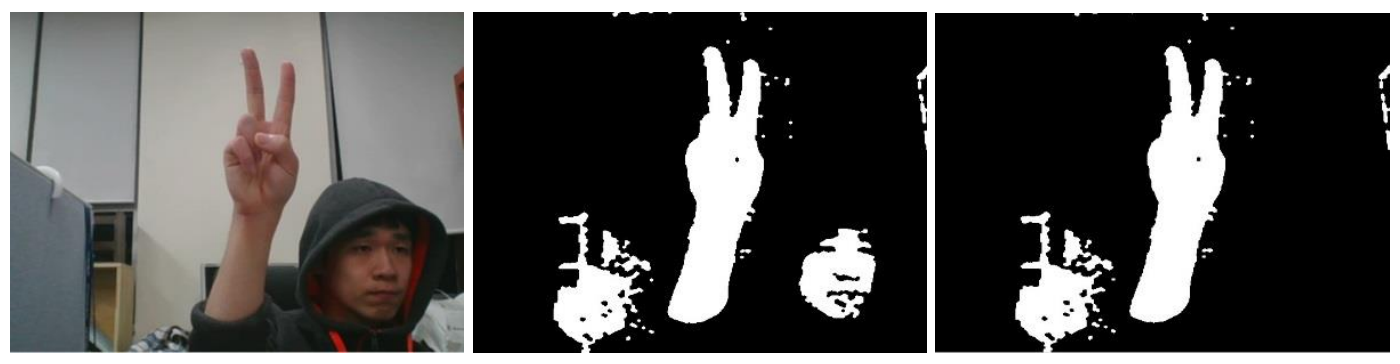

Figure 1. An Example of Skin Color Detection and the Result of Removing Face

As you can see in Figure 1, it's very common that hand candidates cân be extracted along with wrists and forearms. The wrists and forearms mus increase the difficulty in recognition process and degrade the accuracy of recognition. In sucb cases, we should collect larger training samples to consider a great varlety of shapes of hands. Figure 2 shows some examples of segmented hand candidates. As you can see in the figure, hand candidates contain not only hands but also wrists and/or foreams

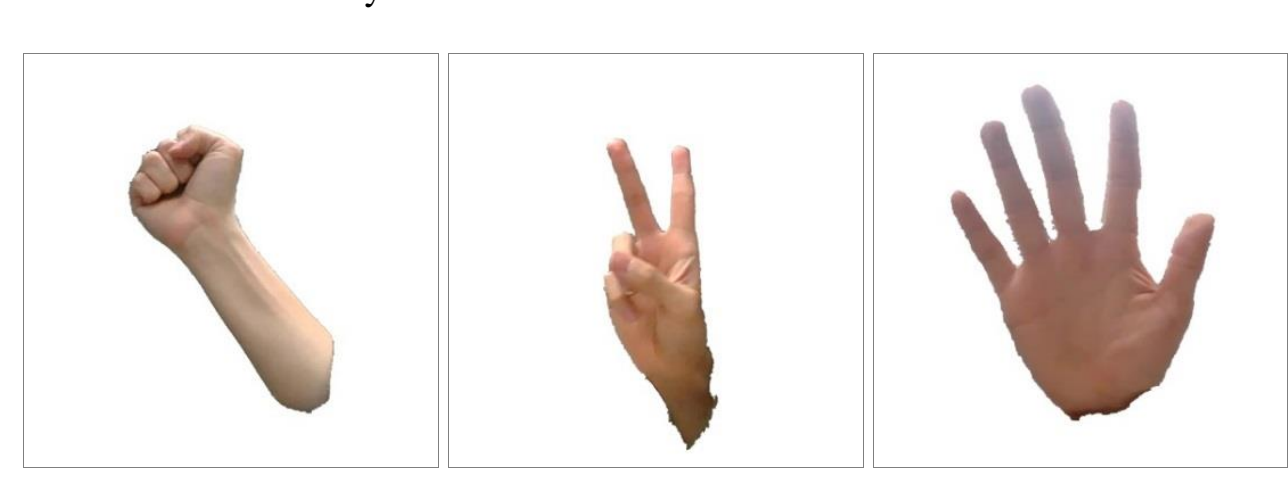

Fiqure 2. Some Examples of Segmented Hand Candidates

We should remove wrists and forearms from hand candidates for improving the accuracy in the recognition. The removing process consists of two steps - finding locations of forearms in which the wrists are placed in the hand candidates and detecting boundaries between hands and wrists. To finding the location of forearm in a hand candidae, we use distance transformation [18] that calculates the distance between foreground pixels and their closest background ones. We can find most inner or centroid position of the candidate by the distance transformation. And then we calculate a minimum distance $\left(d_{\text {min }}\right)$ between the centroid and contour pixels of the candidate. We draw a circle centered in the centroid and with radius of $\alpha \cdot d_{\min }$ where $\alpha$ is a predefined constant. Next, we count the number of objects that meet with circumference of the circle and calculate length of the objects. Along the main direction of the arm, a hand is located at end of the candidate and a wrist is on the opposite. Location of forearms can be decided by following rules. When one side of the candidate satisfy rule 1 , then hand located in the side of the candidate. Rule 2 is for forearm location.

1. If there are more than two objects that meet circumference of the circle 
2. If length of object is within range of $\beta \cdot 2 d_{\min }<d<\beta \cdot 2 d_{\min }$. When length of all objects in two direction are fallen within the range, we draw a circle again with increasing $\alpha$ and repeat to check rule 2 .

Figure 3 shows an example of a hand extraction result. Figure 3(a) and 3(c) are a segmented hand candidate by using skin color detection and a hand detection result by removing the forearm from the candidate, respectively. In Figure 3(b), you can see the centroid of the object. Rectangle of $\boldsymbol{0}$ in Figure 3(b) is a minimum bounding box along the main direction (2 in the Figure 3(b)) of an arm. Distance in 3 shows a minimum distance between centroid and contour pixels of the object.

Next step to remove the forearm from the candidate to detect the boundary between a hand and a wrist. First, a line is selected. The line is vertical to the main direction of the arm and passes a point that is apart from centroid by $d_{\min }$ toward the wrist of the arm (Figure 3(b)). Then curvature is calculated in two pixels that is located at the end of each side of the line and also in the neighbor pixels of the end pixels. We selected two pixels from candidate pixels in each side of the line, which are located in the location Mith maximum curvature. We decided a line that is a boundary between a hand and a wrist by following the three rules below. Regions that are located outside of the line are removed from the candidate.

1. When the maximum curvature values of two points exceed the predefined threshold, select a line that connects two points.

2. When just one value of curvature exceeds the threshold, select a line that is vertical to the main direction of the arm and passes the point of the maximum curvature.

3. When neither points exceed the threshold, select a line ghat is vertical to the main direction of the arm and passes a point that is apart from centroid by $d_{\min }$ toward the wrist of the arm.

You can see the case corresponding torule 2 in Frgure 3(b). The line 4 in Figure 3(b) shows direction and also distance rom the centroid toward main direction of the arm. The line $\boldsymbol{5}$ is a line that is a boundary between the hand and the wrist. So we can remove the region below the line. Figure 3(c) shows the result after removing the wrist.

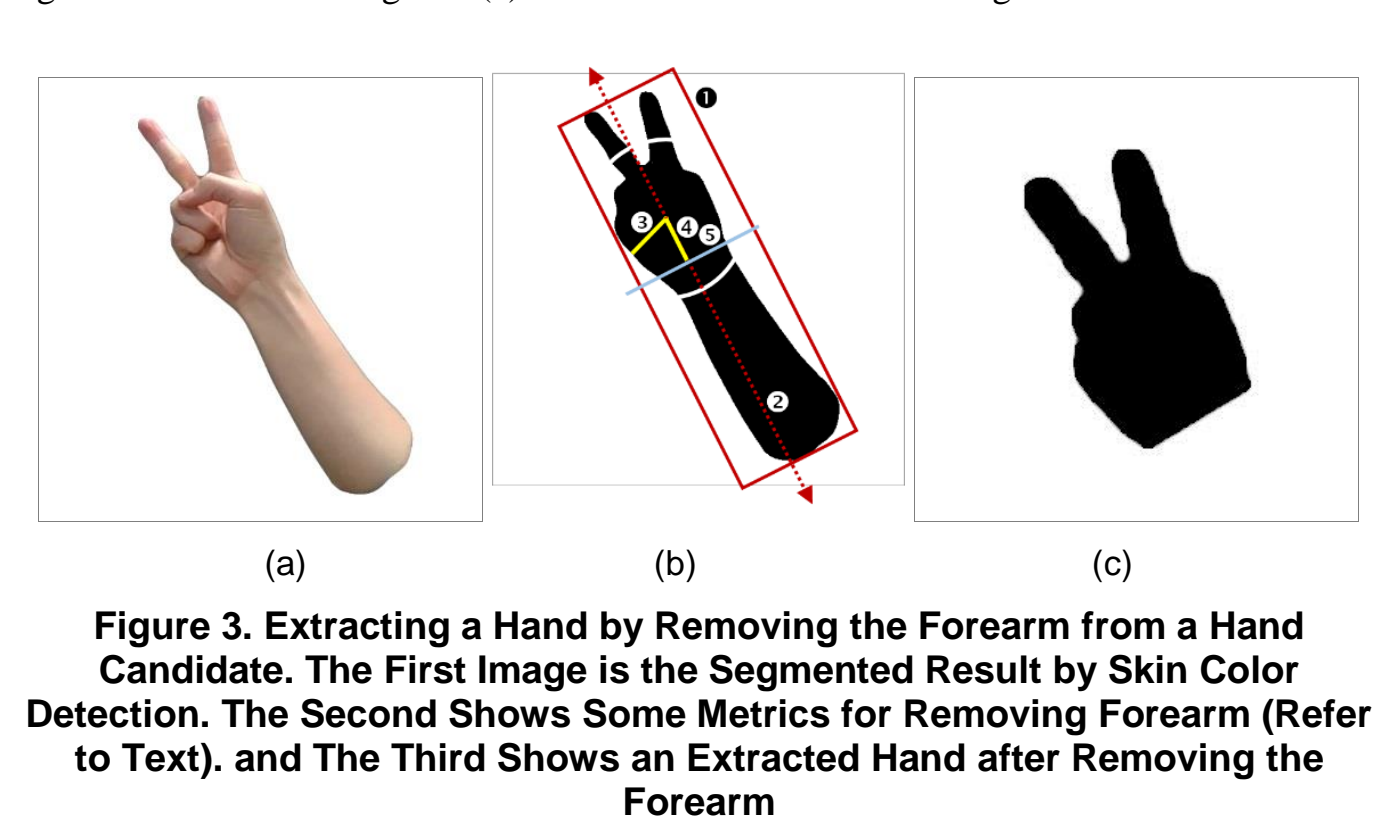

\subsection{Classification of Hand Shapes}

After localizing hands from hand candidates, we calculate feature vectors that represent hand shapes effectively. Two kinds of moment invariants are used to represent the shapes 
- Hu and Zernike moment. Then we create a new feature by fusing the two existing features.

Two kinds of moment invariants and their fusion are used as like in our previous study [2] and we verify the performance of these features in hand shape recognition. In the previous study, we supposed that hands could be extracted without any connected forearms but it is a very illogical condition. In this paper, we improve the performance by removing forearms from hand candidates. Also we try to reduce dimension of feature vectors. Dimension of feature vectors in $\mathrm{Hu}$ moments is fixed but one of Zernike moments is increased rapidly with higher orders. So it needs to reduce the dimension of the feature vectors.

Principal component analysis (PCA) can transform a set of data into a set of linearly uncorrelated components. So we can use just fewer principal components than original feature vectors to train classifier or to test unknown patterns. Principal component with larger Eigen-values can contribute in the classification much more than smaller ones

We train artificial neural network by using four types of feature vectors - two basic moment invariants of $\mathrm{Hu}$ and Zernike, fusion of them and transformed version of Zernike by PCA. We compare the performance of the four types of the vectors. And we analyze the effect on accuracy according to orders in Zernike moments and to numbers of principal components.

We use seven kinds of classes of hand shapes to train artificial netral network. Figure 4 shows selected examples of the classes. Four of seven classes are for rock-paperscissors game and the others for robot control.

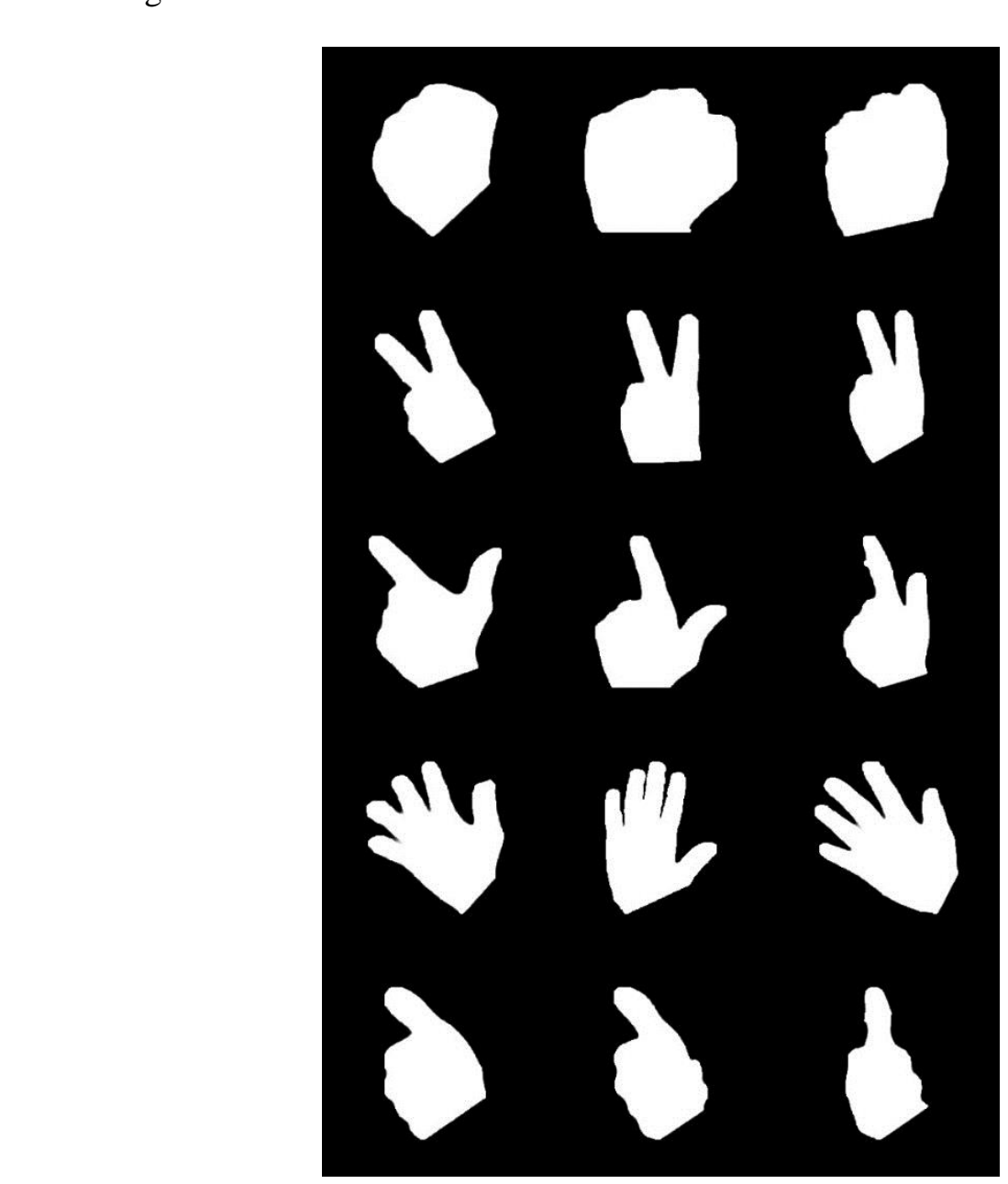




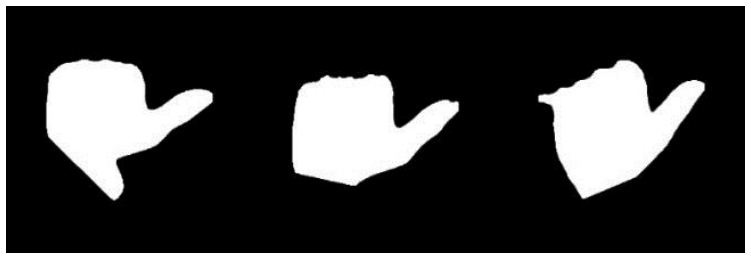

Figure 4. Selected Examples of Training Images of Classes. We Use Seven Classes to Train Hand Shapes. Four Classes among them are for RockPaper-Scissors Game and the others for Robot Control

\subsection{Classification of Digits}

We try to classify another set of data i.e., digits from vehicle license plate. We don't consider characters in the plate because character recognition is beyond the ability of this study. Figure 5 shows selected examples of train images of the ten classes in digits dataset.

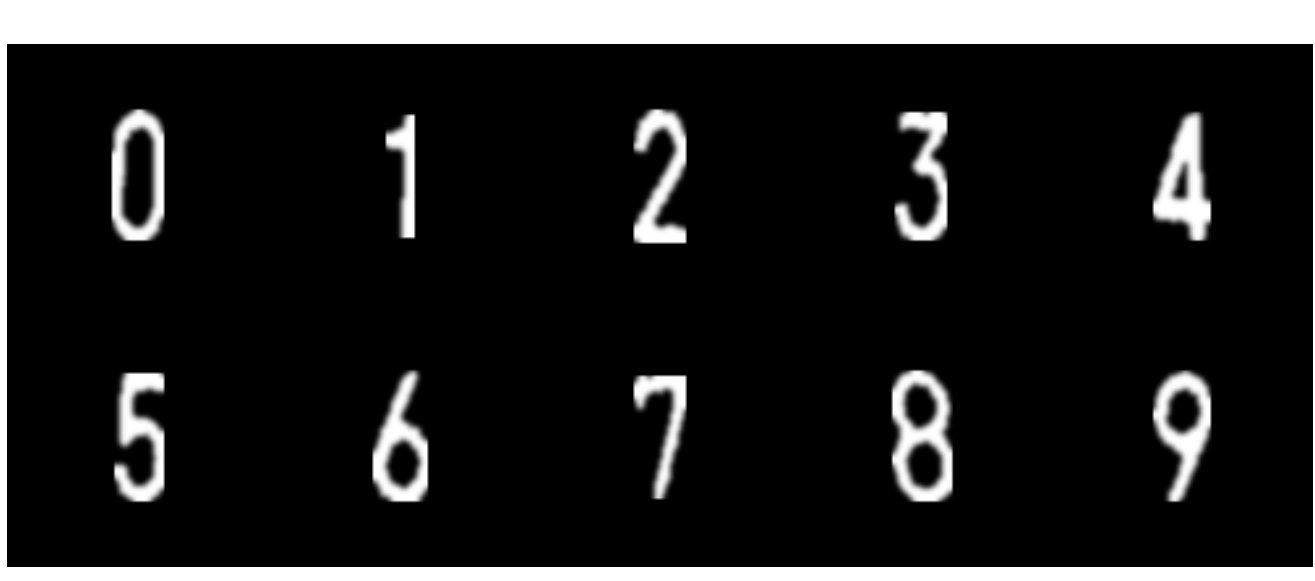

Figure 5. Selected Examples of Training Images of Classes in Digits

We use the same features set with hand shape recognition but except PCA version of Zernike moments. So we train artificial neural network by using three types of feature vectors. We also compare the performance of the three types of vectors according to orders in Zernike moments

\section{Experimental Results}

We classified hand shapes into seven classes, and digits into ten classes. For evaluating accuracy, we captured the hand images by using webcam and the images of vehicle license plate by using infrared camera. We segmented digits from the plate images manually. Hand dataset consists of 630 color images from three different persons. Each hand shape class consists of 90 images of size $300 \times 300$ from segmented input images of size $280 \times 720$. We assessed recognition accuracy with $n$-fold cross validation. In the case of digit recognition, we trained neural network classifier by using 1000 images consisting of 100 images per each digit class and evaluated performance with new 500 images.

The accuracy of $\mathrm{Hu}$ moments in recognition of hand shapes and digits are $87.5 \%$ and $79.0 \%$, respectively. Figure 6 show recognition accuracy according to orders in Zernike moments for two kinds of dataset. The number of attribute in Zernike moments according to orders shown in Table 1. The accuracy is increasing continuously until the order of 10 but it does not show the salient improvement above the order. Figure 6(a) is for hand shape and 6(b) for digits. If we use order of 3 or above in Zernike moment for recognition, Zernike moment and the fusion with $\mathrm{Hu}$ outperform the $\mathrm{Hu}$ moments. We can assess that 
fused features show better accuracy against Zernike moments and in the lower orders especially. When we use Zernike moments for recognition in hand dataset, the best accuracy is $97.1 \%$ with order of 9 . And it's $98.4 \%$ with order of 20 in digit dataset.

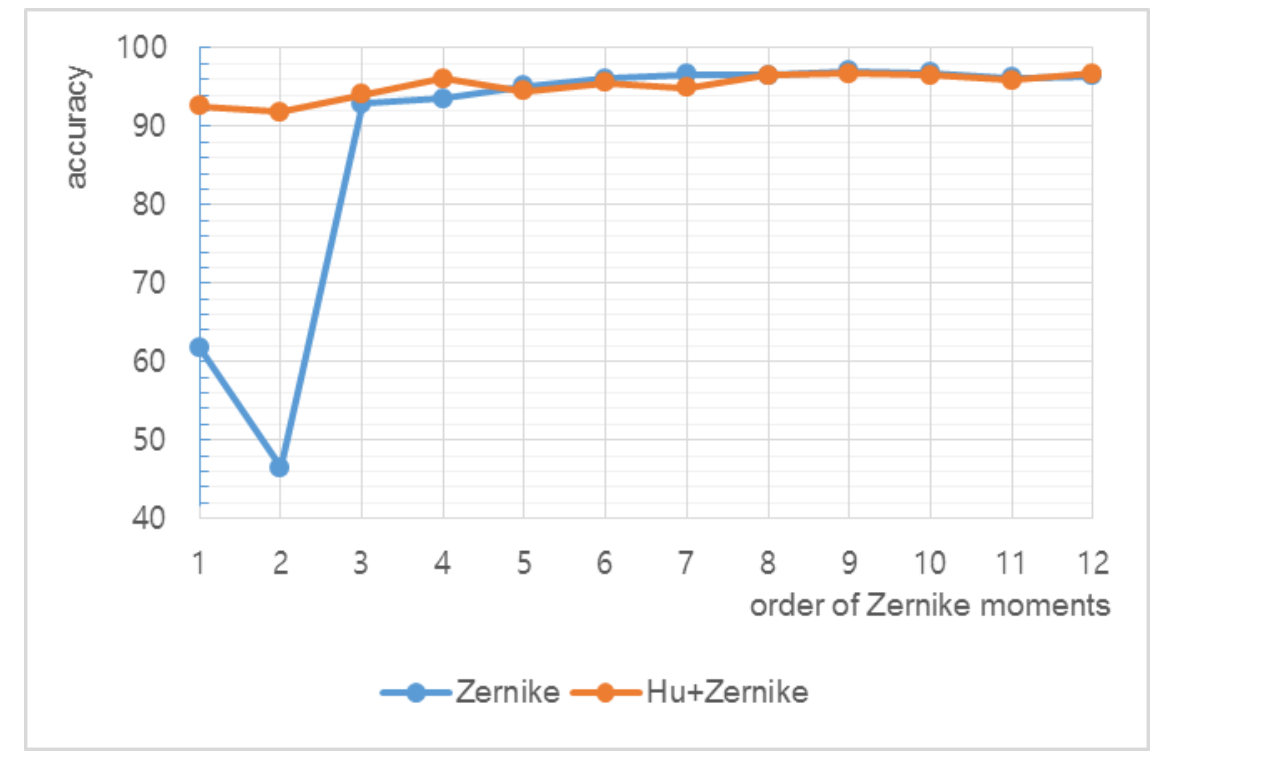

(a) Recogition Accuracy in Hand Shape Recognition

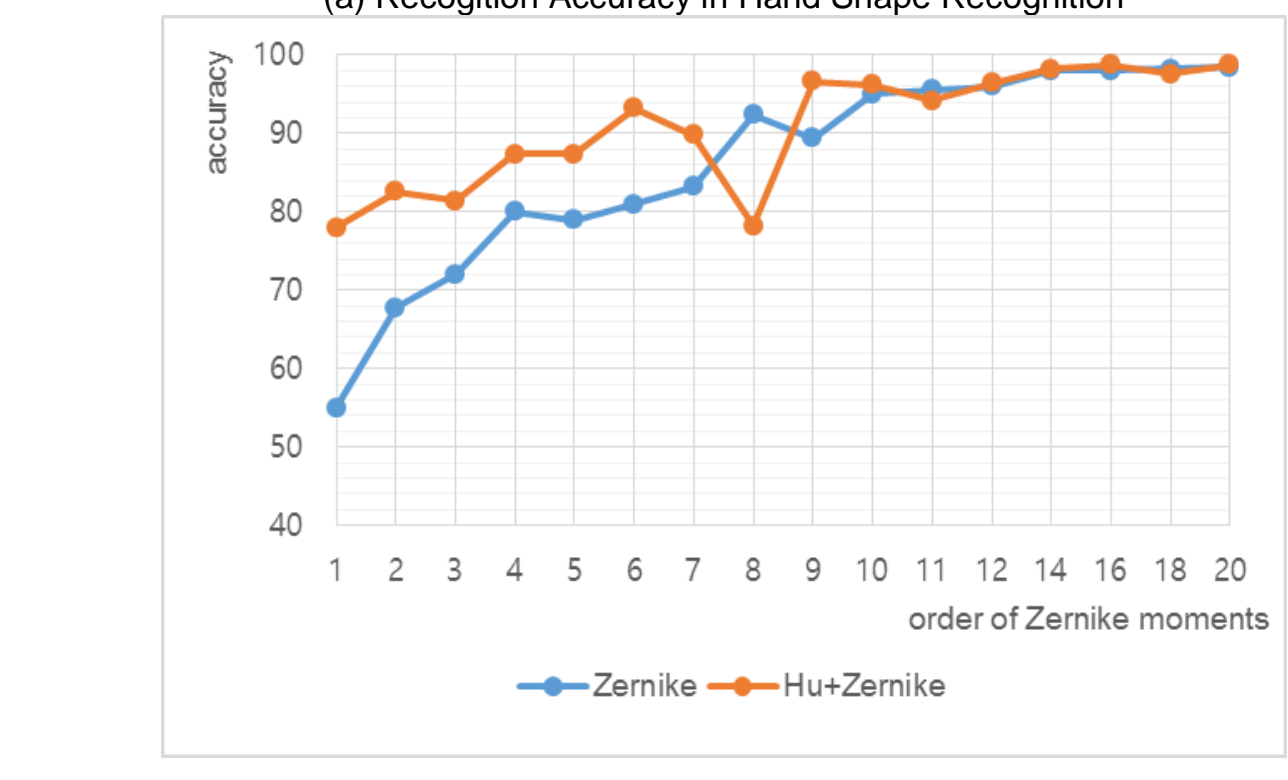

Figure 6. Recognition Accuracy According to Orders of Zernike Moments by Using Feature Vectors of Zernike Moments 
Table 1. Number of Attributes According to Orders in Zernike Moments

\begin{tabular}{|l|l|l|l|l|l|l|l|l|l|l|l|l|l|l|l|l|l|l|l|l|}
\hline & $\mathbf{1}$ & $\mathbf{2}$ & $\mathbf{3}$ & $\mathbf{4}$ & $\mathbf{5}$ & $\mathbf{6}$ & $\mathbf{7}$ & $\mathbf{8}$ & $\mathbf{9}$ & $\mathbf{1 0}$ & $\mathbf{1 1}$ & $\mathbf{1 2}$ & $\mathbf{1 3}$ & $\mathbf{1 4}$ & $\mathbf{1 5}$ & $\mathbf{1 6}$ & $\mathbf{1 7}$ & $\mathbf{1 8}$ & $\mathbf{1 9}$ & $\mathbf{2 0}$ \\
\hline$\#$ & 2 & 4 & 6 & 8 & 12 & 16 & 20 & 25 & 30 & 36 & 42 & 49 & 56 & 64 & 72 & 81 & 90 & 100 & 110 & 121 \\
\hline
\end{tabular}

Table 2, 3 and 4 show the classification results on hand dataset. The results are represented with confusion matrices. Class 0 in the tables corresponds to hand shape of rock and the others are same sequences in Figure 4. In Table 3 and 4, the order of Zernike moments is 9 which show the best results in Figure 6(a).

Table 2. Classification Results of Hand Shapes in Confusion Matrix Based on Hu Moments

\begin{tabular}{|c|c|c|c|c|c|c|c|}
\hline Pred. & 0 & 1 & 2 & 3 & 4 & 5 & 6 \\
\hline 0 & 89 & 1 & & & & & \\
\hline 1 & & 87 & 3 & & & & \\
\hline 2 & 1 & 4 & 69 & 15 & 1 & & \\
\hline 3 & 1 & & 1 & 88 & & & \\
\hline 4 & & & & & 89 & 2 & \\
\hline 5 & & & 7 & 1 & 1 & 75 & 6 \\
\hline 6 & & 2 & 9 & & 2 & 22 & 55 \\
\hline
\end{tabular}

Table 3. Classification Results of Hand Shapes In Confusion Matrix Based on Zernike Moments

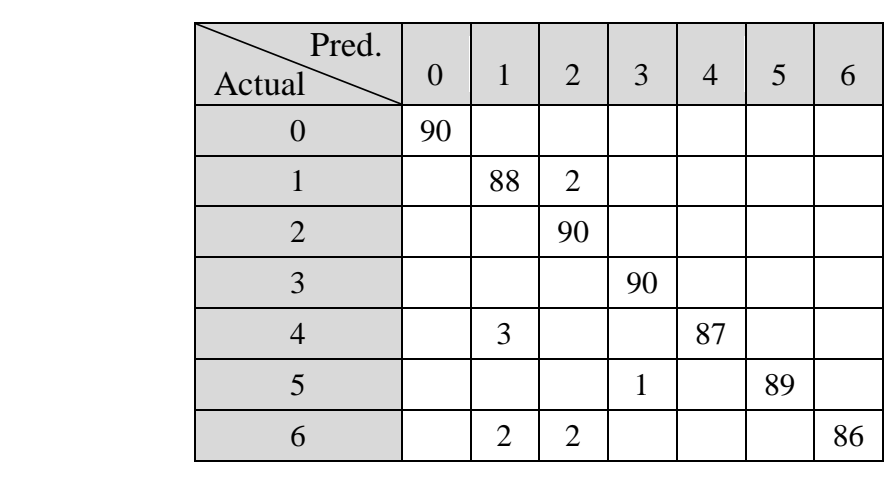

Table 4. Classification Results of Hand Shapes in Confusion Matrix Based on the Fusion of Hu and Zernike Moments

\begin{tabular}{|c|c|c|c|c|c|c|c|}
\hline Pred. & 0 & 1 & 2 & 3 & 4 & 5 & 6 \\
\hline 0 & 89 & & & & & 1 & \\
\hline 1 & & 87 & & 3 & & & \\
\hline 2 & & & 90 & & & & \\
\hline 3 & & & & 89 & & 1 & \\
\hline 4 & & 1 & & & 89 & & \\
\hline 5 & & & & 2 & & 88 & \\
\hline 6 & & 1 & & & 3 & 2 & 84 \\
\hline
\end{tabular}


Table 5, 6 and 7 show the classification results on digits dataset. The results are represented with confusion matrices. Class 0 in the tables corresponds to digit 0 . In Table 6 and 7, the order of Zernike moments is 20, show the best results in Figure 6(b). In Table 5 , you can see that digit 6 and 9 can't be distinguished because two digits are very similar in their shape and differ in the direction.

Table 5. Classification Results of Digits in Confusion Matrix Based on Hu Moments

\begin{tabular}{|c|c|c|c|c|c|c|c|c|c|c|}
\hline Actual Pred. & 0 & 1 & 2 & 3 & 4 & 5 & 6 & 7 & 8 & 9 \\
\hline 0 & 39 & & & & & 3 & & & 8 & \\
\hline 1 & & 50 & & & & & & & & \\
\hline 2 & & 4 & 34 & 12 & & & & & & \\
\hline 3 & & & 10 & 40 & & & & & & \\
\hline 4 & & & & 1 & 49 & & & & & \\
\hline 5 & 1 & & 2 & 1 & & 43 & & & 3 & \\
\hline 6 & & & & & & & 49 & 1 & & \\
\hline 7 & & & & & & & & 50 & \\
\hline 8 & 8 & & & & & & & & 41 \\
\hline 9 & & & & & & & 49 & 1 & \\
\hline
\end{tabular}

Table 6. Classification Results of Digits in Confusion Matrix Based on Zernike Moments?

\begin{tabular}{|r|r|r|r|r|r|r|r|r|r|r|}
\hline Actual Pred. & 0 & & 2 & 3 & 4 & 5 & 6 & 7 & 8 & 9 \\
\hline 0 & 50 & & & 6 & & & & & \\
\hline 1 & & 50 & & & & & & & & \\
\hline 2 & & & 49 & 1 & & & & & & \\
\hline 5 & & & 50 & & & & & & \\
\hline 4 & & & & 50 & & & & & \\
\hline 5 & & & & & & 50 & & & & \\
\hline 6 & 1 & & & & & & & & 49 & \\
\hline 9 & & & & & & & 5 & & & 45 \\
\hline
\end{tabular}


Table 7. Classification Results of Digits in Confusion Matrix Based on the Fusion of Hu and Zernike Moments

\begin{tabular}{|c|c|c|c|c|c|c|c|c|c|c|}
\hline Actual Pred. & 0 & 1 & 2 & 3 & 4 & 5 & 6 & 7 & 8 & 9 \\
\hline 0 & 50 & & & & & & & & & \\
\hline 1 & & 50 & & & & & & & & \\
\hline 2 & & & 49 & 1 & & & & & & \\
\hline 3 & & & & 50 & & & & & & \\
\hline 4 & & & & & 50 & & & & & \\
\hline 5 & & & & & & 50 & & & & \\
\hline 6 & & & & & & & 50 & & & \\
\hline 7 & & & & & & & & 50 & & \\
\hline 8 & & & & & & & & & 50 & \\
\hline 9 & & & & & & & 5 & & & 45 \\
\hline
\end{tabular}

We also evaluate the performance of PCA on Zernike moments. Figure 7 shows the recognition accuracy according to the number of principal components in the hand dataset. Recognition result with Zernike moments show best accuracy in the order of 9. With order of 9, there are 30 of properties. We assess the accuracy of recognition by increasing the number of principal components that are used for training the classifier. The accuracy is $94.66 \%$ when we use just 7 of 30 components, and the value is kept with more components. When we use 7 components $879 \%$ of tôta energy is used. So we can reduce the number of feature for training classifier and testing the performance.

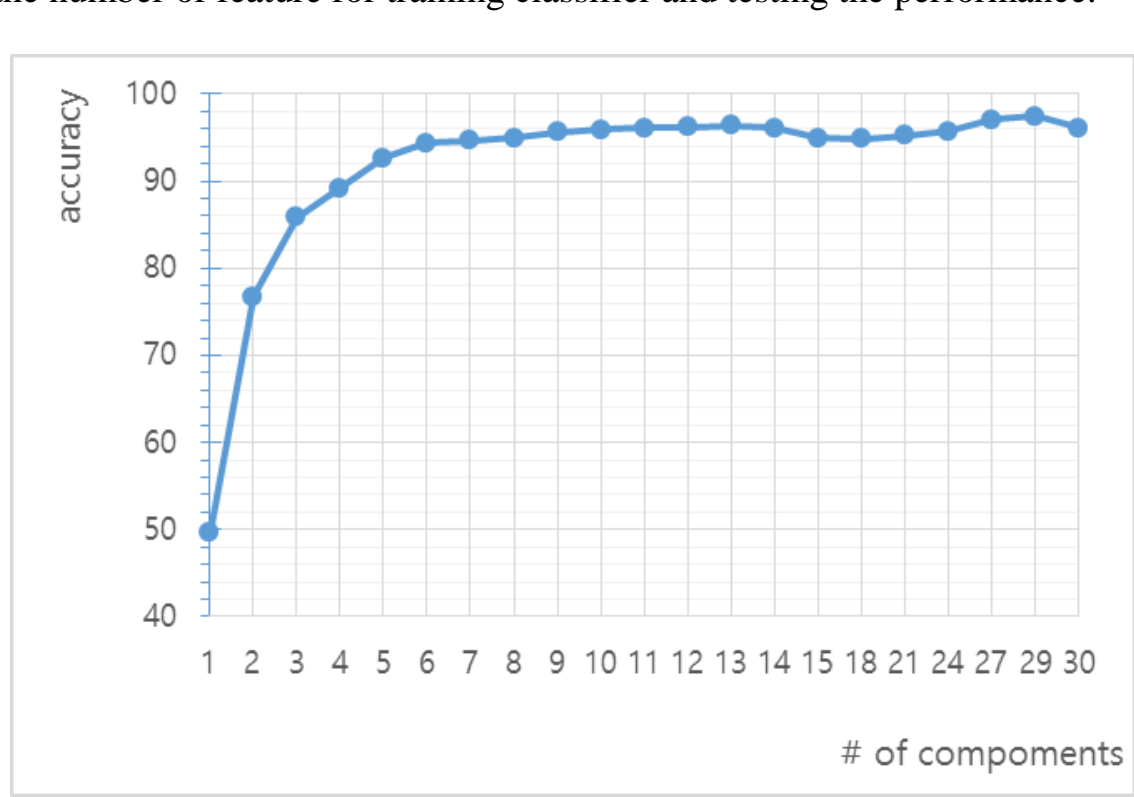

Figure 7. Recognition Accuracy According to the Number of Principal Components with Zernike Moments

\section{Conclusion}

We explored the contribution of moment invariants in pattern recognition of hand shapes and digits. Hu and Zernike moments could show good performance but were not enough to satisfy the requirement of some applications. Hu moments gave a little low accuracy in digit dataset and Zernike moments were with the lower orders. We could increase the accuracy by fusing the $\mathrm{Hu}$ and Zernike moments. The fused feature could 
give improved accuracy especially in the lower orders of Zernike moments. By applying the PCA on the Zernike moments, we could reduce the dimension of feature vector without degrading the ability of Zernike moments.

\section{Acknowledgements}

This paper was supported by Research Fund, Kumoh National Institute of Technology.

\section{References}

[1] S. Kim and J. J. Cheon, "Strokes Separation from Hand Motion Trajectories", 4th International Conference on Circuits, Control, Communication, Electricity, Electronics, Energy, System, Signal and Simulation, vol. 51, no. 1, (2014), pp. 64-67.

[2] S. Leem, H, Jeong, Y. Lee and S. Kim, "A Robust Hand Gesture Recognition Using Combined Moment Invariants in Hand Shape", 4th International Conference on Interdisciplinary Research Theory and Technology, (2016), pp. 89-94.

[3] P. Priyal and P. K. Bora, "A Study on Static Hand Gesture Recognition using Moments", In 'I Conf. on Signal Processing and Communications (SPCOM), (2010).

[4] V. Kumar, G. C. Nandi and R. Kala, "An invariant-based approach to static Hand-Gesture Recognition", 18th Int'l Conf. on Artificial Reality and Telexistence, (2008).

[5] Z. Zou, P. Premaratne, R. Monaragala, N. Bandara, and M. Premaratne, "Dynamic Hand Gesture Recognition System using Moment Invariants", 5th International Conference on Information and Automation for Sustainability (ICIAFs), (2010).

[6] T. Wang and S. Liao, "Chinese Character Recognition by Zernike Moments", 2014 International Conference on Audio, Language and Image Processing (ICALIP), (2014).

[7] W. H. Wong, W. C. Siu, and K. M. Lam, "Generation of moment invariants and their uses for character recognition”, Pattern Recognition Letters, vol. 16, no 2, (1995), pR 115-123.

[8] Y. D. Khan, S. A. Khan, F. Ahmad, and S. Islam, "Iris Recognition Using Image Moments and k-Means Algorithm", the Scientific World Journal, rol. 2014, (2014).

[9] J. Haddadnia, K. Faez and P. Moallem, Neural network based face recognition with moment invariants", International Conference on lmage Processing, (2001), pp. 1018-1021.

[10] M. Liu and J. X. Mao, "Traffic Signs Recognition Based on Affine Invariant Hu's Moment Features", Applied Mechanics and Materials, vol. 321-324 (2013), pp. 945-949

[11] R. Mukundan and K. R. Ramakrishnan, "Fasfcomputation of Legendre and Zernike moments", Pattern Recognition, vol. 28, no. (1995), pp. 1433-1442.

[12] C. W. Chonga, P. Raveendranb, and R. Mukundanc, "A comparative analysis of algorithms for fast computation of Zềrnike moments”, Pattern Recognition, vol. 36, no. 3, (2003), pp. 731-742.

[13] P. Viola and M. Jones, "Rapid Object Detection using a Boosted Cascade of Simple Features", Conf. on CVPR, (2001).

[14] T. Flusser, T Suk and B. Zitova, "Moments and moment invariants in pattern recognition", Wiley, (2009).

[15] M. J. Jones and J. M. Rehg, "Statistical Color Models with Application to Skin Detection", Int. J. of Computer Vision, y1 46, ho. 1, (2002), pp. 81-96.

[16] S. Y. Kim and $F$ P. Ko, "Skin Color Detection Using PCA-based Color Representation", International Journal of Mulitimedia and Ubiquitous Engineering, vol. 9, no. 10, (2014), pp. 231-242.

[17] A. Mittal, A. Zisserman, and P. H. S. Torr, "Hand detection using multiple proposals", British Machine Vision Conference, (2011).

[18] R. Kimme N. Kiryati and A. M. Bruckstein, "Distance maps and weighted distance transforms", Journal of Mathematical Imaging and Vision, Special Issue on Topology and Geometry in Computer Vision, vol. 6, (1996), pp. 223-233.
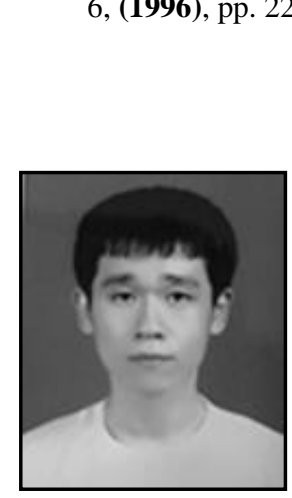

\section{Authors}

Hyunseok Jeong, Mail: asdfgh25@naver.com. Research Interest: Image processing, Information Communication. He is a fourth-year undergraduate at Kumoh National Institute of Technology and major in computer engineering. 


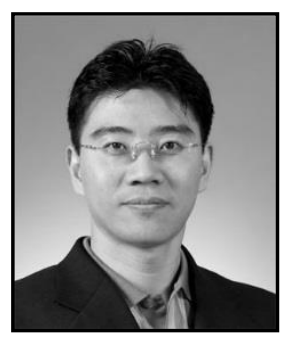

Sungyoung Kim, Mail: sykim@kumoh.ac.kr. Research Interest: Image processing, Pattern recognition, Multimedia information processing. He received $\mathrm{Ph}$. D. in computer engineering from Pusan National University, Korea in 2004. From 2004, he is a professor in dept. of computer engineering at Kumoh National Institute of Technology.

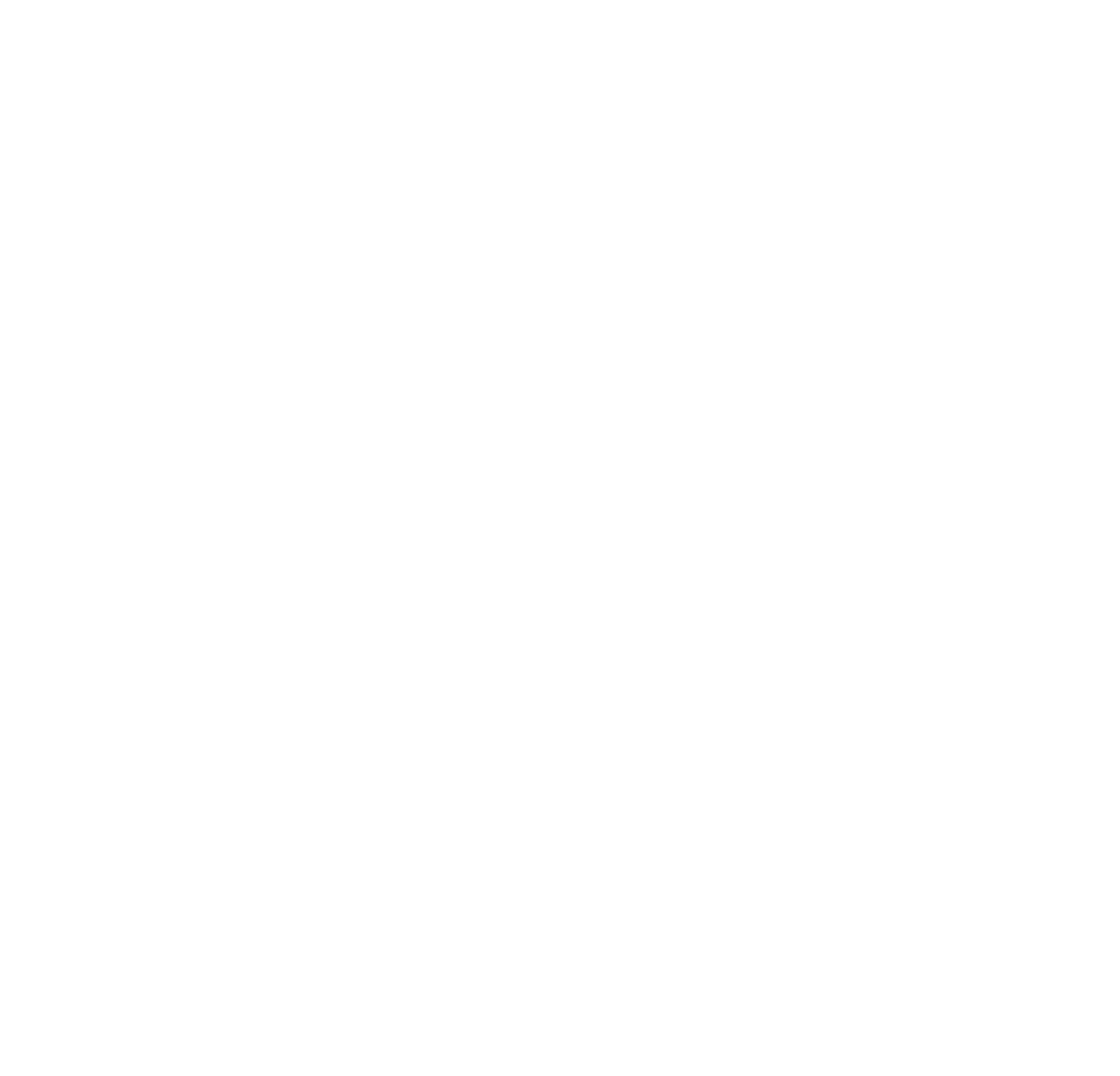


International Journal of Multimedia and Ubiquitous Engineering

Vol.11, No.11 (2016)

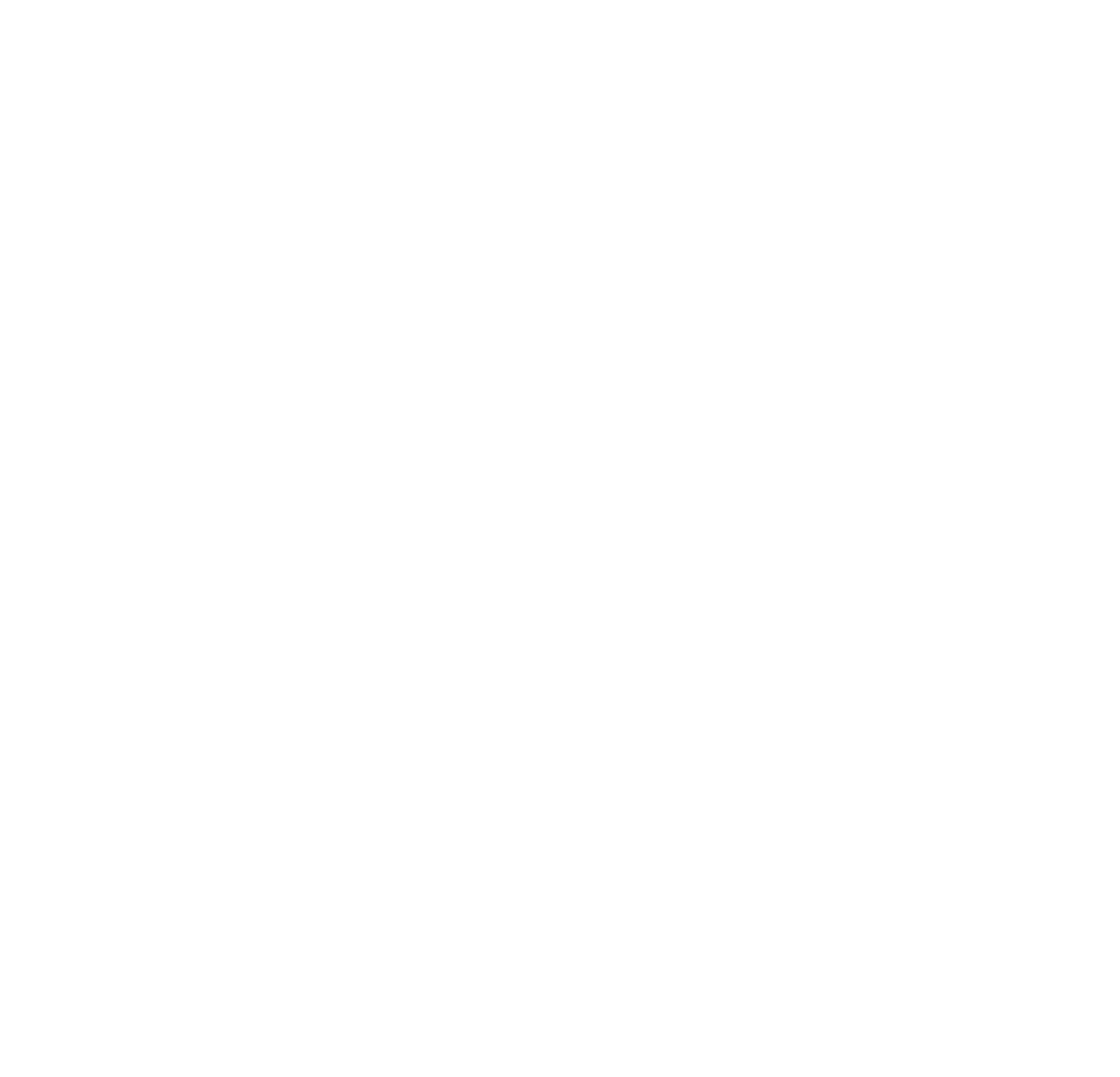

